\title{
Ergebnisse: Studien(fach)wahlen im Kontext von sozialem Milieu und Geschlecht
}

\subsection{Milieuspezifika und Fallsynopse}

Die analysierten Wege in das Bauingenieurwesen und die Soziale Arbeit zeigen, wie Studien(fach)wahlen eingebettet sind in die vergeschlechtlichte milieuspezifische Lebensführung und wie die antizipierte Passung zu einem Studienfach und einem Beruf in diesem Zusammenspiel entsteht. Je nach vergeschlechtlichter milieuspezifischer Habitualisierung werden die Handlungsmöglichkeiten auf dem Weg in einen Beruf kanalisiert bzw. erweitert, bestimmte Dispositionen werden gestärkt und andere in den Hintergrund gerückt. Was das Verhältnis der Fälle zueinander angeht, haben sich Ähnlichkeiten und Kontraste in den Analysen bereits angedeutet und bilden sich ebenso in ihrer Verortung im Modell sozialer Milieus ab (vgl. Abbildung 6.1). Nach der ausführlichen Einzelanalyse sollen die vergeschlechtlichten Milieuspezifika der Fälle nun noch einmal dargestellt und in einer Fallsynopse ${ }^{1}$ (vgl. Tabelle 7.1) zusammengetragen werden. Dann werden sie entsprechend ihrer Nähe zueinander geclustert und die mit den Studien(fach)wahlen verbundenen sozialen Logiken im Sinne typologischer Muster zusammengefasst. Um die herausgearbeiteten kulturellen Passungsverhältnisse zu fundieren, werden die Erkenntnisse der Fachkulturforschung zur Sozialpädagogik und dem Ingenieurbereich herangezogen (insbesondere Zinnecker 2004; Sander/Weckerth 2017; auch Haffner 2014).

\footnotetext{
${ }^{1}$ In dieser Fallsynopse wurde bewusst darauf verzichtet, die Habitusdimension von Geschlecht in einer gesonderten Spalte darzustellen, da sie jede Ebene des Falls und des Habitus prägt und davon nicht getrennt werden kann. Dabei erweisen sich die Vorstellungen von Familiengründung und -organisation der Befragten als besonders relevanter milieu- und geschlechtsspezifische Aspekt der Antizipation eines Berufs, sodass diese gesondert dargestellt werden.
} 
Sonjas Fall ist geprägt von der Selbstverständlichkeit eines Studiums im Kontext ihrer , akademischen Technikfamilie - eine Tradition, die für Sonja handlungsleitend ist. Sonja weist eine hohe fachliche Affinität zur Mathematik und den damit verbundenen ,logischen' Inhalten auf, die in ihre Herkunftsfamilie eingebettet sind. Dass bereits ihre Mutter als Frau ein technisches Studium absolviert hat und nun in einem entsprechenden Beruf tätig ist, scheint die Selbstverständlichkeit von Sonjas grundsätzlicher fachlicher Ausrichtung noch zu verstärken. Zudem ist Sonjas Blick auf das Studium geprägt durch das Hintergrundwissen ihrer Mutter darüber, wie es ist, als Frau ein technisches Fach zu studieren. Sonjas Weg in das Studium war ein geradliniger und strategischer, der allerdings durch vergeschlechtlichte Abdrängungseffekte - etwa im Unterricht oder durch den väterlichen Rat - hin zu einem (universitären) Mathematikstudium begrenzt wurde. Der Gründung einer eigenen Familie kommt für Sonja eine hohe Bedeutung zu, sie thematisiert sie von sich aus. Dabei antizipiert sie die schwerpunktmäßige Übernahme der Erziehungsarbeit und will dies als eigene Entscheidung jenseits gesellschaftlicher Zuschreibungen verstanden wissen. Es zeigen sich bei ihr auch ausgrenzende Wahrnehmungsmuster sowie eine starke Orientierung an ihr bekannten Strukturen und Stabilität.

Tabea entpuppt sich in weiten Teilen als Kontrastfall zu Sonja. Die unterschiedlichen Einflüsse von ihrem Vater und ihrer Mutter können als Einflüsse unterschiedlicher Milieutraditionen verstanden werden, die den beruflichen Orientierungsprozess für Tabea erschweren ${ }^{2}$ : Von den väterlichen Bildungsplänen für Tabea - ausgerichtet an Status, Prestige und der Kumulation von kulturellem Kapital - grenzt sie sich vehement ab, weil diese in den Umgebungen ihres Aufwachsens für sie nicht umsetzbar bzw. erstrebenswert sind. Stattdessen identifiziert sie sich mit dem für sie positiv besetzten ,niedrigen Umfeld“, in dem sie bei ihrer Mutter aufgewachsen ist und bezeichnet das soziale Umfeld ihres Wohnprojekts als „Familie“. Ihre Orientierung hin zur Sozialen Arbeit wird somit maßgeblich dadurch gestärkt, dass sie nach der Trennung ihrer Eltern bei ihrer Mutter aufwächst. Dabei ist Tabeas Weg zum Studium durch umfangreiche Suchbewegungen geprägt und verläuft über mehrere Stationen: vom abstrakten Universitätsstudium über die bodenständige Schreinerinnenlehre bis zur Sozialen Arbeit, mit der sie sich über den expliziten mütterlichen Rat gegen dieses prekäre Beschäftigungsfeld hinwegsetzt. Tabeas geringe Passung zum Bauingenieurwesen fußt nicht (nur) auf einer fachlichen Distanz, sondern auch auf einer

\footnotetext{
${ }^{2}$ Was auf weiteren Forschungsbedarf im Rahmen des Milieuansatzes hinweist, denn die Mechanismen der Paarbildung und der möglicherweise recht unterschiedliche habitusspezifische Einfluss von Elternteilen in der Erziehung sind bislang nicht untersucht.
} 
milieuspezifischen Distanz zu der ingenieuralen Fachkultur und den dortigen Werten, Vermittlungsformen und Anforderungen (,Formeln eintrichtern“). Auch von Materialismus, Prestigedenken und Karrierestreben distanziert sich Tabea stark. Ihr Verständnis von der Sozialen Arbeit ist geprägt von einer distinktiven Betonung des Unkonventionellen (es ist kein „Büro-0815-Job“) und einem idealistischen Blick auf den sozialarbeiterischen Beruf als egalitäre, gesellschaftsformende Instanz. Eine Familie zu gründen, ist Teil ihrer Zukunftsvorstellungen und zusammen mit ihrer Abgrenzung von einem Fokus auf die Erwerbsarbeit führt das dazu, dass sie die Familienarbeit übernehmen und dieses (aus ihrer Sicht) ,Privileg' nicht ihrem Partner überlassen möchte. So antizipiert auch Tabea die Ausübung der Erziehungsarbeit schwerpunktmäßig auf ihrer Seite.

Auch bei Achim sind Prestige- und Karriereorientierung oder materielles Denken nicht handlungsleitend, wobei er sich nicht so ausdrücklich davon abgrenzt, wie es bei Tabea der Fall ist. Außerdem finden sich in Achims Umfeld keine akademischen Vorbilder $^{3}$ und auch die Soziale Arbeit ist in seiner Familie nicht vertreten. Und bei ihm zeichnen sich in Kindheit und Jugend Notwendigkeitserfahrungen größeren Ausmaßes ab als bei Tabea. In der Folge setzt sich Achim überschaubare, für ihn realistische und eher mittelfristige Ziele, eine langfristige Lebensplanung mit festen Zielen kommt für ihn nicht in Frage. Seine alleinerziehende Mutter konnte ihn während der Schulzeit nur begrenzt unterstützen und zugleich durchlief er eine Schullaufbahn ohne Leistungsdruck von außen oder feste elterliche Erwartungen. Stattdessen wurde er durch sein soziales Umfeld ergebnisoffen unterstützt - ob er nun „Anwalt“, „Tanzlehrer“ oder „Architekt“ werden wollte. Die Orientierung hin zur Sozialen Arbeit ist dadurch geprägt, dass fürsorglich konnotierte Tätigkeiten jenseits von finanziellem Gewinndenken auch ausgeübt durch seine Mutter und seinen Bruder - für Achim positiv besetzt sind. Auch hier spielt also eine gewisse familiale fachliche Tradition eine Rolle für die Studien(fach)wahl. Zugleich scheint für Achim eine tendenziell egalitäre Aufteilung von Haushaltstätigkeiten selbstverständlich zu sein, denn diese Aufteilung wurde von seiner Mutter vorgelebt und gefördert. In der Sozialen Arbeit sieht Achim die Möglichkeit, den „Alltag“ mit den Klient*innen zu ,gestalten“. Mit diesem Berufsziel eng verknüpft ist Achims Streben nach möglichst großer Handlungsfreiheit. Diese Handlungsfreiheiten wurden ihm schon von seinem sozialen

\footnotetext{
${ }^{3}$ Im Folgenden wird statt des Begriffs der ,Vorbilder“ auch der der „Orientierungspersonen“ benutzt, da der Vorbild-Begriff im Sinne einer aktiven, zielgerichteten Nachahmung verstanden werden kann. Von dieser Deutung, die sich so auch im Interviewmaterial nicht widerspiegelt, soll durch die Einführung einer alternativen Bezeichnung im Ergebnisteil der Arbeit Abstand genommen werden. Da beide Begriffe jedoch einander ähnlich sind und der Vorbild-Begriff in der Forschung üblich, wird auf ihn nicht ganz verzichtet.
} 
Umfeld ermöglicht und vermittelt, ebenso der leitende Wert von Rückhalt aus der Gemeinschaft. Handlungsfreiheit, Offenheit und eine eher mittelfristige Lebensplanung prägen auch Achims Vorstellung von Familiengründung, in welcher er zwar eine positiv besetzte Vaterrolle antizipiert, die für ihn aber kein festes Ziel ist (,Alles kann, nix muss“). Zusammengenommen zeigen sich in Achims Falls damit vergleichsweise wenig einschränkende binäre Einflüsse dahingehend, was ,ein Mann' tun kann, soll oder muss.

Der Kontrastfall zu Achim findet sich bei Michael, denn Michael ist wie kein anderer Fall von Disziplin und Erfolgsstreben geprägt sowie einer patriarchalen Familienorganisation. Hinter Michaels Wunsch nach „Unabhängigkeit“ wirken Handlungsorientierungen hin zu Status(absicherung) durch eigene Leistung, die sich auch in seinen Herkunftserfahrungen widerspiegeln. Die starke Erfolgs- und Erwerbsorientierung wird familiär patriarchal weitergegeben - vom Vater an die Söhne und von Michael an seinen Neffen. Entlang der Geschlechtsrollen ist Michaels Familie eine funktional organisierte unternehmerische Einheit, in der Frauen in die gemeinsame Selbstständigkeit eingebunden, aber ebenso für die Familienarbeit zuständig sind ${ }^{4}$. Michaels Weg in das Studium ist mit Umwegen verlaufen, die er im Nachhinein aber als Erfolge konstruiert. Das Bauingenieurwesen knüpft so an seine Maurermeisterausbildung an und soll das ,zweite Standbein" neben der Automobilbranche und Fundament der zukünftigen Selbstständigkeit mit seiner Partnerin sein, die vor allem auf finanziellen Gewinn und materiellen Besitz abzielt. Michaels Weg in das Bauingenieurwesen ist fachlich damit eher von Zufällen begleitet, wie seiner Vermittlung in eine Maurerausbildung. Zugleich ist die Wahl eines aussichtsreichen technischen Studiums bei ihm dahingehend angelegt, dass er das Bauingenieurwesen für sich als Business deutet, das für ihn die Erfüllung von Status- und Erfolgsansprüchen verspricht.

Dominic lässt sich als dritter männlich-sozialisierter Fall zwischen den beiden anderen verorten: Auch bei ihm zeigt sich eine starke Orientierung an Gemeinschaft - ähnlich Achim (und Tabea), durch die emotionaler Rückhalt und Freundschaften für ihn handlungsleitend sind. Dominics Schullaufbahn ist zudem geprägt von einem kooperativen Umgang in der Familie, der sich nicht am Erreichen eines möglichst hohen Bildungsabschlusses, sondern an Dominics Wünschen und Ressourcen ausrichtet. Sein Weg in die Soziale Arbeit ist vor diesem Hintergrund zum einen geprägt von seinem unerfüllten Wunsch des Polizeiberufs und

\footnotetext{
${ }^{4}$ Die Arbeitsteilung und Organisation von Michaels Herkunftsfamilie erinnert an die bis zum 18. Jahrhundert vorherrschende Form des ,ganzen Hauses“ (Brunner 1994 [1966]: 76), im Rahmen derer Haushalt und Betrieb untrennbar miteinander verwoben waren.
} 
zum anderen dem Abbruch seiner Gymnasiallaufbahn. Die Wahl der Fachrichtung für seine Fachhochschulreife trifft Dominic auch hier gemeinschaftlich und kristallisiert im Austausch mit seinem sozialen Umfeld für sich heraus, welches Studienfach das richtige für ihn ist - denn „das Soziale“ läge ihm doch. Sein Verständnis der Sozialen Arbeit wiederum ist nicht so offen und egalitär wie es sich bei Achim andeutet, sondern auch von dem Wunsch nach Anerkennung und einer gewissen Machtposition verbunden. Er antizipiert eine berufliche Tätigkeit, in der er die Welt seiner Klient*innen wieder , in Ordnung ' bringt. In Dominics Fall ist es also keine familial-fachliche Disposition hin zu Fürsorgetätigkeiten (wie bei Achim), durch die er sich der Sozialen Arbeit annähert. Stattdessen gerät diese Berufsmöglichkeit erst dadurch in seinen Blick, dass er kein Polizist werden kann. Gestärkt wird diese Berufsperspektive durch sein Verständnis von der Sozialen Arbeit als helfende, aber eben auch ,ordnende " Instanz sowie seine familial angelegten kooperativen und gemeinschaftlichen Habituszüge. In dieses Bild fügen sich auch seine Vorstellungen von Familiengründung ein: Eine eigene Familie zu gründen, ist für Dominic ein festes Ziel und auch hier kommt der Wert von Gemeinschaft zum Tragen, denn er stuft „Familienzusammenhalt“ als höchste Priorität ein. Durchaus heteronormativ strukturiert - aber weitaus egalitärer als bei Michael - ist seine beteiligende Vorstellung von Familienarbeit.

Unter den anderen Fällen von künftigen Sozialarbeiter*innen sticht Rebecca wiederum besonders heraus - ihr Fall verdeutlicht, dass vielfältige milieuspezifische Wege in dieses Studium führen können und dass Dispositionen hin zu Gemeinschaft oder Egalität dabei nicht die maßgeblichen sein müssen ${ }^{5}$. So ist Rebecca Michael gar nicht unähnlich: Auch ihr Habitus ist geprägt von einem hohen $\mathrm{Ma}$ an Leistungsbereitschaft und Disziplin, um die Schullaufbahn und ihren Studienwunsch zu verwirklichen. Allerdings ist bei ihr ein stärkerer „Kampf“ erkennbar als bei Michael. Rebecca deutet die Soziale Arbeit gemäß ihrer Disposition hin zum Streben nach einem prestigeträchtigen Beruf um und der (nicht immer erfüllte) Aufstiegswunsch bei ihr und ihrer Familie wird deutlich. Rebeccas individualistische Leistungs- und Aufstiegsorientierung schlägt sich so auch in ihrer sendungsbewussten Vorstellung von ihrem Traumberuf der Kinder- und Jugendtherapeutin nieder (,Wer kämpft, kann den Aufstieg schaffen'). Zugleich zeigen sich weniger gewinnorientierte Handlungsorientierungen als bei Michael, denn für Rebecca sind materielle Ziele wenig bedeutsam und stattdessen spielen Freude und Sinn im Beruf eine größere Rolle. Auch zeigt sich bei ihr wie bei Dominic ein gewisser Stellenwert von Macht. Auffällig in

${ }^{5}$ Gleichwohl diese Dispositionen die Passung zur Sozialen Arbeit entscheidend bestärken mögen im Sinne einer Nähe zur dominanten Fachkultur. 
Rebeccas Berufsorientierung ist ihr Blick auf männliche Orientierungspersonen und Unterstützer wie ihren Vater und ihren Partner. Rebeccas Fokus auf erwerbsbezogene Leistung geht für sie mit einer stärkeren Orientierung an männlichen Personen einher. Was die Familiengründung und -organisation angeht, antizipiert Rebecca zwar vorreflexiv die Zuständigkeit für die Familienarbeit, grenzt sich davon allerdings zugleich $a b$ und wertet sie $a b-z u$ groß ist ihr Fokus auf die Erwerbsarbeit.

Eine ganz andere Färbung hat der letzte sozialarbeiterische Fall von Jennifer: Bei ihrem Weg in den Beruf ist - ähnlich Tabea, Achim und Dominic - keine Orientierung an Prestige erkennbar und auch bei ihr nimmt die Gemeinschaft einen zentralen Platz ein. Sie hat frühe Erfahrungen in einer fürsorglichen Rolle gemacht und familiäre Verantwortung für ihre Geschwister übernommen (bzw. übernehmen müssen). Bis heute sieht sie sich in einer fürsorglichen Rolle, die ihr auch Freude bereitet. Im Gegensatz zu Achim scheint in Jennifers Fall die Nähe zu Fürsorge matriarchal weitergegeben zu werden - denn außer ihrer Mutter war sie scheinbar die Einzige, die in der Familie entsprechende erzieherische Aufgaben übernommen hat. Erfahrungen des Unsteten und der Notwendigkeit prägen Jennifers Aufwachsen und tragen zu ihrem Schulabbruch nach dem Hauptschulabschluss bei. Unterstützt durch ihre Mutter erlernt sie einen qualifizierten Beruf, strebt aber zugleich nach mehr Sinn, Freude, Anerkennung und Autonomie. Gemeinsam mit ihrem Partner zeigt sie Risikobereitschaft und geht strategisch und diszipliniert den Weg in ein Studium, indem sie ihre Fachhochschulreife nachholt. Zugleich zeigen sich bei ihr materielle Bescheidenheit und ein finanzielles Sicherheitsbestreben, das sich auch in ihrem Wunsch nach Familiengründung niederschlägt: Diese antizipiert sie nämlich in Kombination mit einer traditionell-geschlechtlichen Aufgabenteilung aus monetären Gründen, sie ist aber auch Gegenstand von Aushandlungsprozessen zwischen ihr und ihrem Partner.

Schließlich ist auch Hanna eine Bildungsaufsteigerin und nimmt diesen Aufstieg gemeinsam mit einer anderen Person gleicher sozialer Herkunft in Angriff, ihrer Zwillingsschwester. Hanna muss sich jedoch gegen mehr Skepsis seitens ihrer Herkunftsfamilie durchsetzen als Jennifer. Zugleich scheint sie keine vergleichbaren Notwendigkeitserfahrungen gemacht zu haben. Ihr Weg in das Bauingenieurwesen ist fachlich geprägt vom Beruf ihres Vaters, durch den sie Einblicke in die Baubranche erlangt hat. Im Gegensatz zu Sonja thematisiert sie die Bedeutung von Geschlecht nicht selbstständig und geschlechtliche Abdrängungseffekte wie die ,Sonderrolle als Frau' scheinen bislang nicht Teil ihrer bewusst wahrgenommenen Erfahrungen zu sein. Sie erwähnt allerdings auch keine weibliche Orientierungsperson in diesem Berufsbereich und ihre Mutter übt eine andere Tätigkeit aus. Hannas Studienwunsch ist geprägt von ihrem Streben nach 


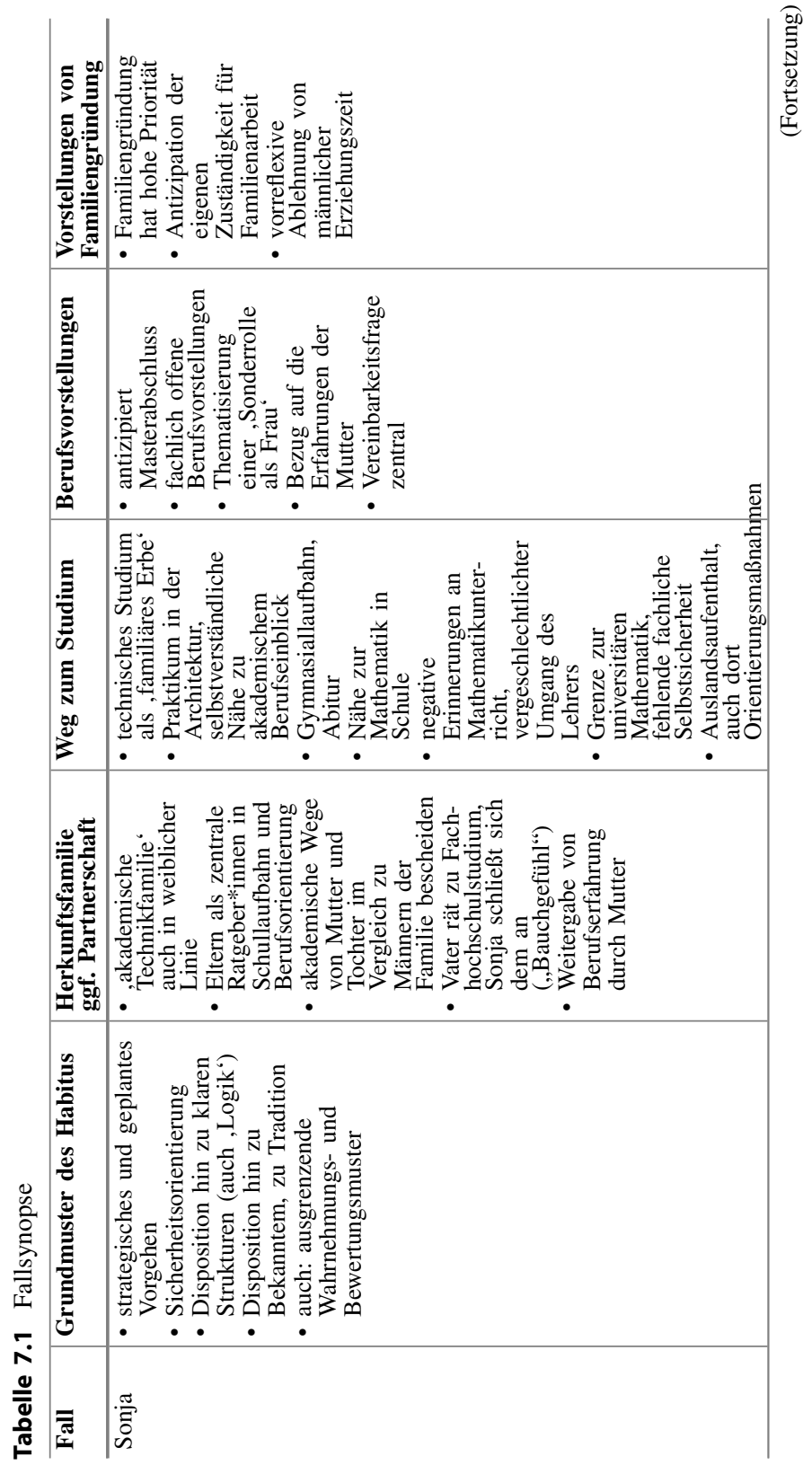




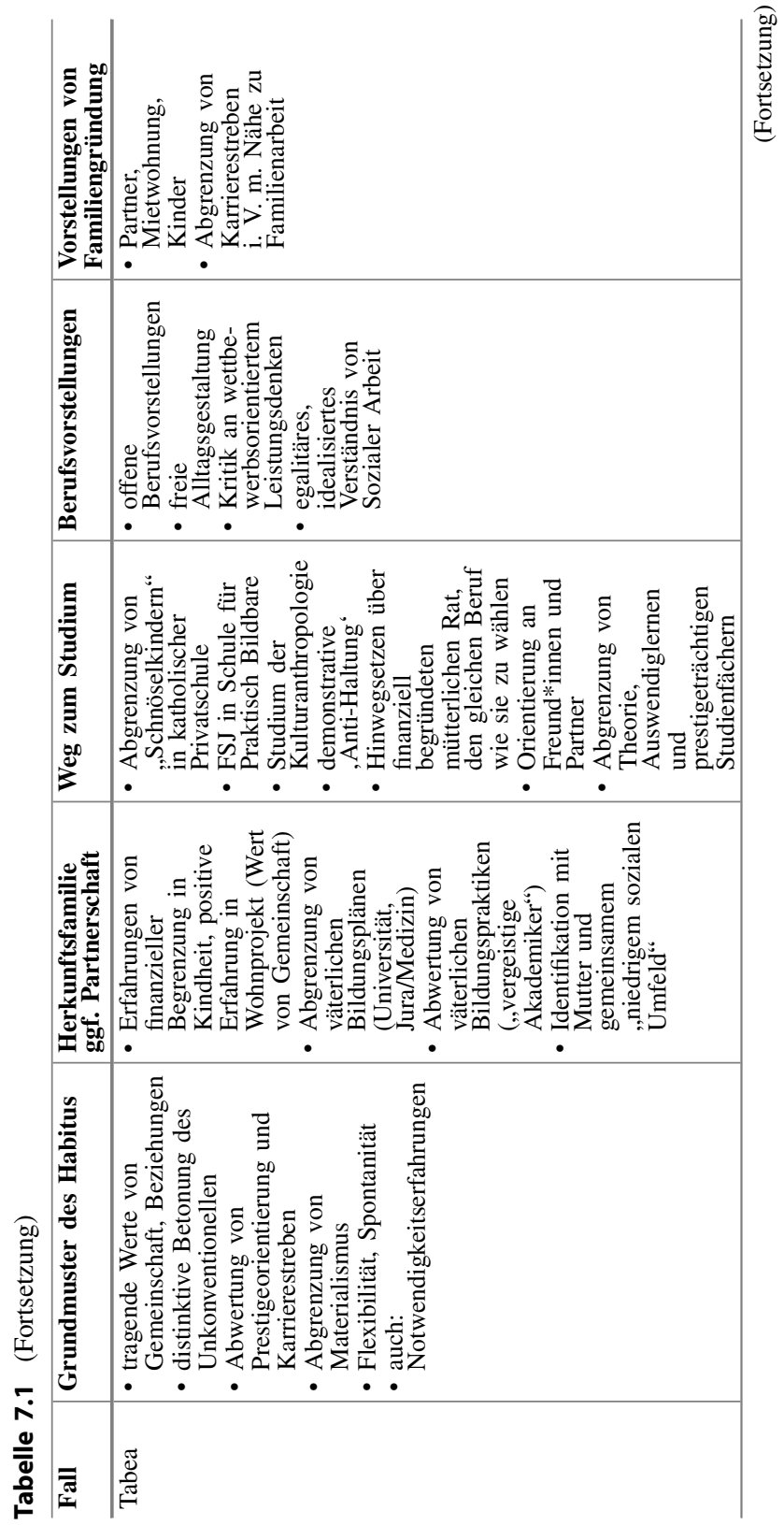




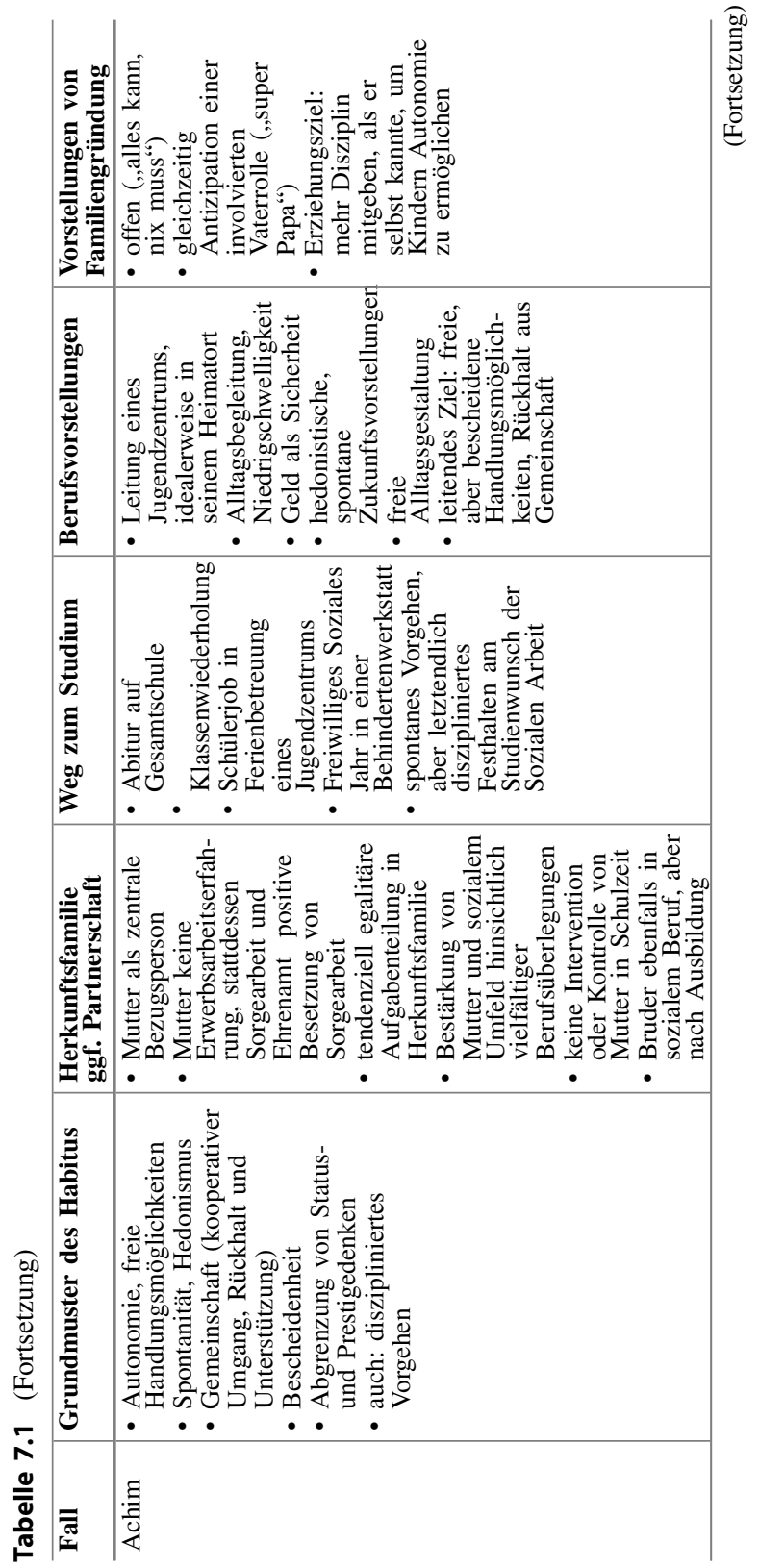




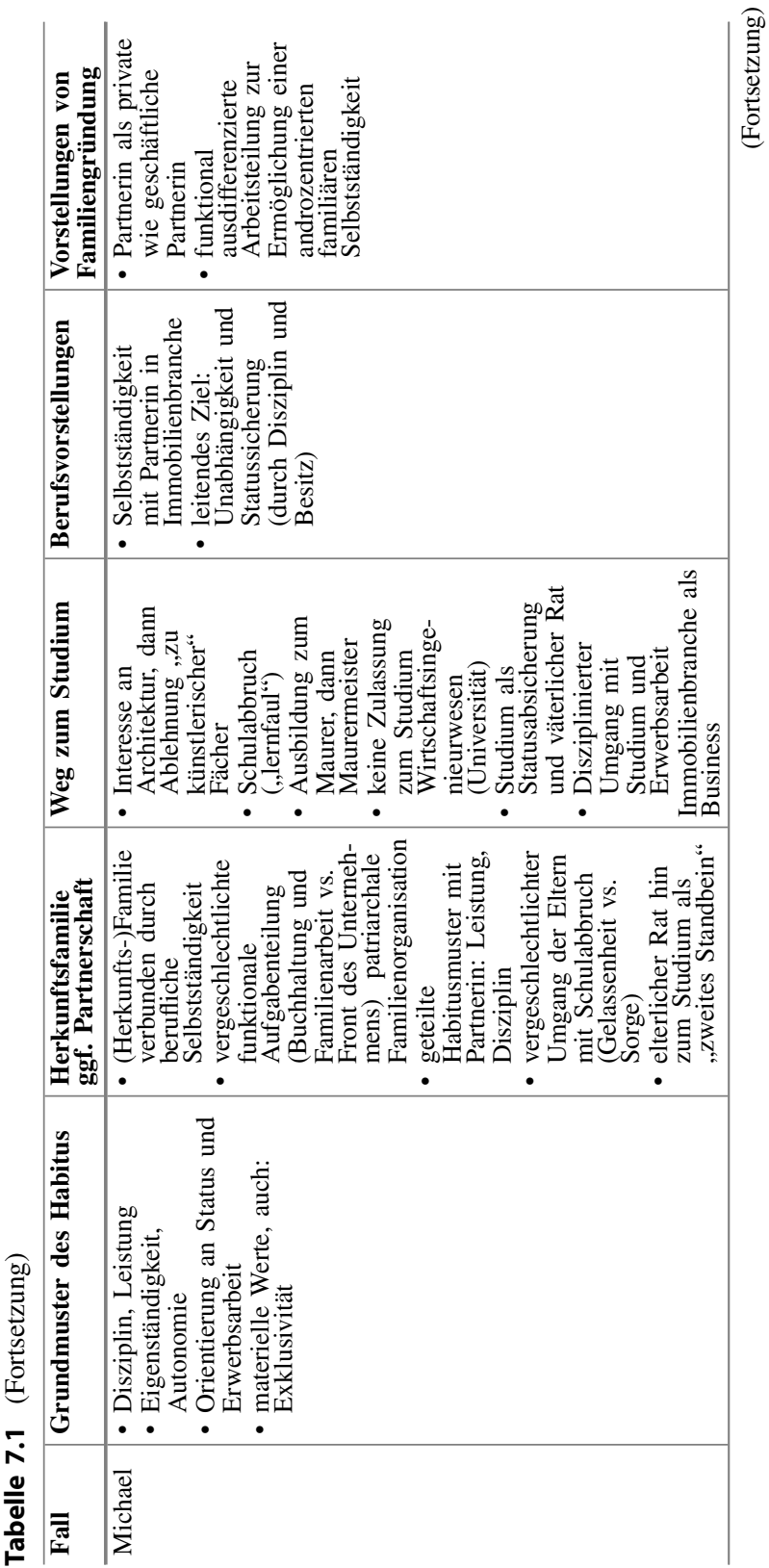




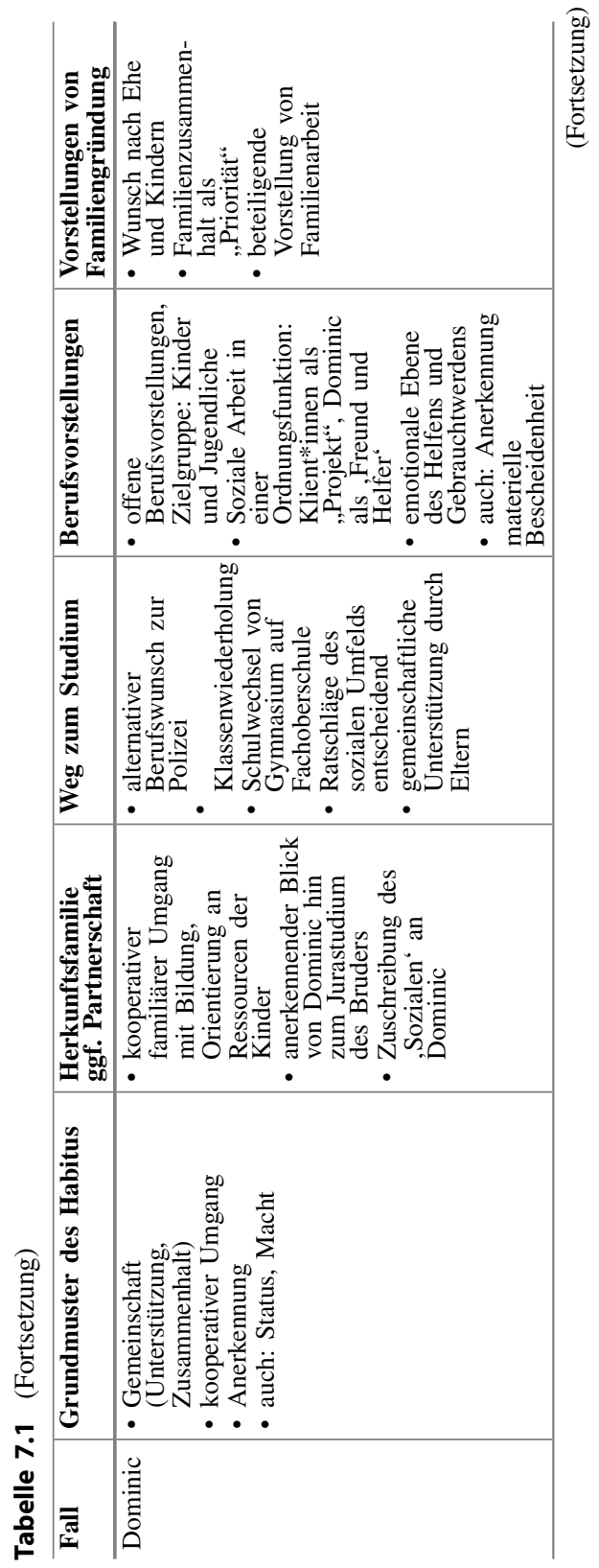




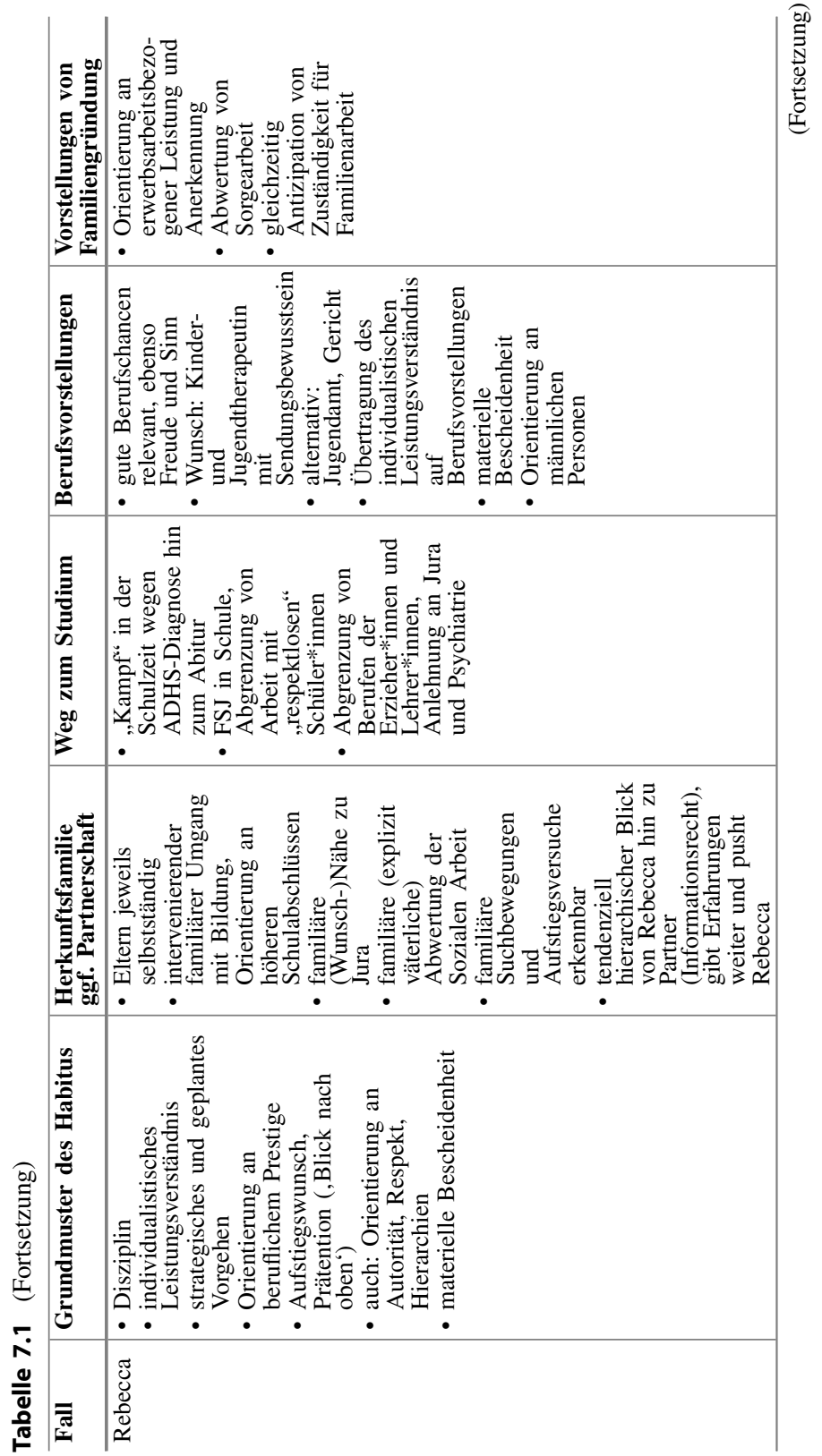




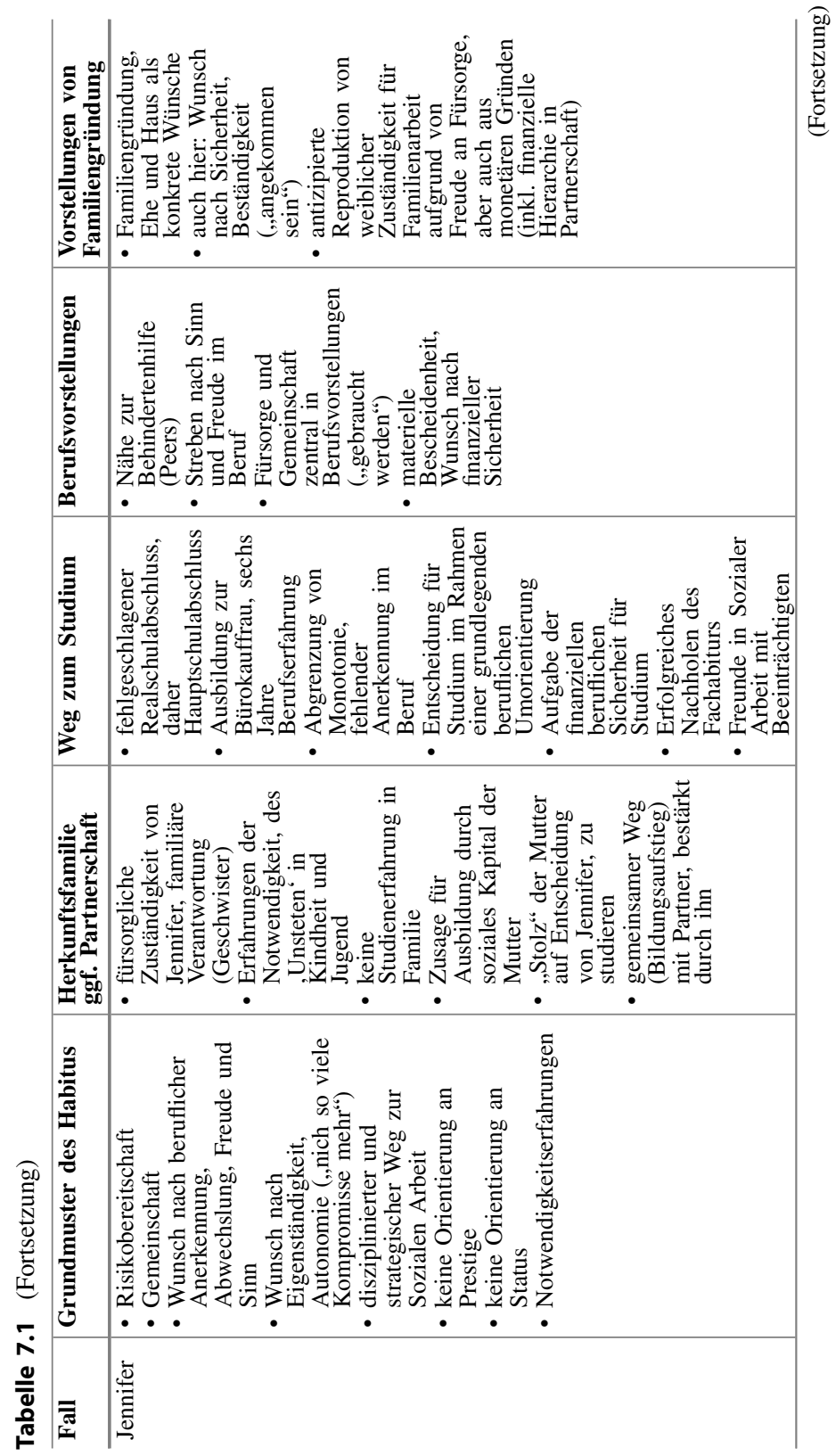




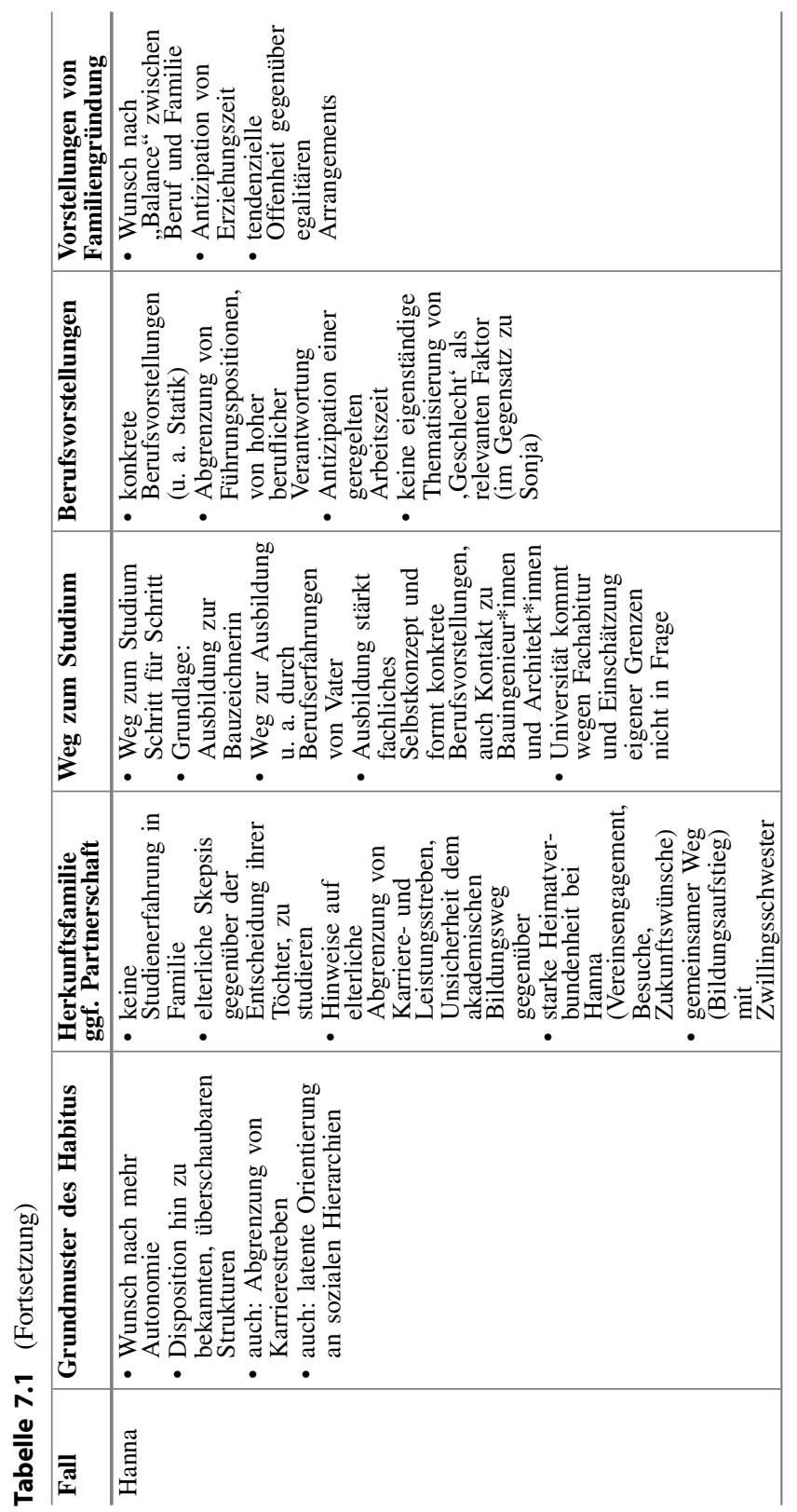


mehr Autonomie und nach einem Aufstieg in der beruflichen Hierarchie hin zu den Bauingenieur*innen und Architekt*innen, die sie als ,vielbeschäftigte Menschen" aus ihrer Ausbildung kennt. Zugleich will Hanna dieses Autonomiestreben nicht ,um jeden Preis“ erfüllen, sondern antizipiert eine Balance zwischen einem geregelten Arbeitsleben als Projektleitung in der Baubranche und der Familiengründung, im Rahmen derer sie zumindest einen Teil der Erziehungsarbeit leisten will. So ist es in Hannas Fall das Zusammenspiel des beruflichen Einblicks über ihren Vater mit den fehlenden bewussten Erfahrungen geschlechtsspezifischer Abdrängung aus dem technischen Bereich - wie Sonja sie erfahren hat - und mit ihrem Wunsch nach Autonomie durch beruflichen Aufstieg, das ihren Weg in das Bauingenieurstudium prägt.

\subsection{Vergeschlechtlichte milieuspezifische Studien(fach)wahlen im Vergleich}

\subsubsection{Studien(fach)wahlen im Kontext von Unkonventionalität und Idealismus}

Das Muster der Studien(fach)wahl, das sich bei Tabeas Weg in die Soziale Arbeit abzeichnet und spezifisch für ihren sozialen Ort ist (s. Abbildung 7.1) lässt sich unter den leitenden Handlungsorientierungen von ,Unkonventionalität und Idealismus“ zusammenfassen. Die Studien(fach)wahl findet in Abgrenzung zu Prestige- und Statusorientierung statt und lässt zugleich distinktive Anteile dahingehend erkennen, dass sie als unkonventionell und altruistisch konstruiert wird (,Die Gesellschaft besser machen'). Es zeichnen sich zudem partielle Notwendigkeitserfahrungen in Kindheit und Jugend $a b$, die ein flexibles und spontanes Vorgehen (mit-)bedingen. Außerdem spielt der leitende Wert von Gemeinschaft, von sozialen Beziehungen und von einem kooperativen, egalitären Umgang miteinander eine Rolle. Materielle Werte werden herabgesetzt.

Geschlechtlich ,erwartungskonforme' Studien(fach)wahlen wie die von Tabea stehen nicht im Fokus der aktuellen Forschung. Umso mehr eröffnet gerade in ihrem Fall die Analyse der milieuspezifischen Muster der Studien(fach)wahl einen bisher verschlossenen Blick auf den Weg einer Frau in einen sozialen Studiengang: In Tabeas Fall bildet ebenfalls ein auf Notwendigkeiten und Gemeinschaft ausgerichteter Habitus die Grundlage für ihre Studien(fach)wahl, im Rahmen dessen sie flexibel und unkonventionell vorgeht. Gleichzeitig schlägt sich bei ihr der Einfluss des akademischen und prestigeorientierten geprägten Vaters nieder, von dem sie sich vehement abzugrenzen sucht, dessen durchaus distinktiver Blick 
auf die Welt sich aber auch bei ihr bis zu einem gewissen Maß niederzuschlagen scheint. Und obwohl Tabea selbst Schreinerin und gerne handwerklich tätig ist, liegt ihr ein technisches Studium besonders fern - denn ihre Passung zur ingenieuralen Fachkultur ist äußerst gering. Dass dort „Freude am zwischenmenschlichen Kontakt, kontroverse Aushandlungs- und Abwägungsprozesse oder kontroverse Diskussionen“ (Sander/Weckerth 2017: 5 f.) - also Orientierungen, die für Tabea mitunter handlungsleitend sind - keine tragende Rolle spielen, entspricht auch Tabeas Gespür und sie sieht sich daher in keinem Fall als künftige Bauingenieurstudentin. Umgekehrt ist ein sozialpädagogisches Studium geprägt von kommunikativ ausgerichteten Lehr- und Lernformen, von der Arbeit in Gruppen und von vergleichsweise wenig Faktenlernen im Gegensatz zu einem überwiegenden Anteil an argumentativem Austausch und Reflexion (Zinnecker 2004) - Aspekte, die an Tabeas Dispositionen gut anschließen. Auch die fachkulturspezifische Haltung von „Wettbewerb zerstört Solidarität“ (Bargel 2007a: 199) spiegelt sich in ihrer Abgrenzung von einem ausgeprägten Erwerbsfokus und Wettbewerbsgedanken wider. Tabeas Abgrenzung von Karrierestreben führt mit Blick auf ihre Zukunftsvorstellungen allerdings nicht zu einer „Erprobung alternativer Lebensweisen“ (ebd.: 180), wie es die Ergebnisse der Fachkulturforschung nahe legen. Stattdessen antizipiert Tabea eine durchaus ,traditionelle“ Aufgabenteilung bei Familiengründung - eine auf betonter Unkonventionalität fußende Studien(fach)wahl muss also keinesfalls mit ,unkonventionellen ' Vorstellungen von Arbeitsteilung verbunden sein. Die Antizipation der Zuständigkeit für die Kindererziehung auf weiblicher Seite wiederum ist in diesem Fall maßgeblich beeinflusst durch die vorliegende Milieuorientierung.

Die hohe Passfähigkeit dieses Musters der Studien(fach)wahl legt nahe, dass allgemein die Dispositionen der weiter links verorteten Milieus, auch des hedonistischen Milieus, tendenziell zur Sozialen Arbeit als Berufsfeld und Studien(fach)wahl ,passen “ - zu diesem Berufsfeld, das weder besonders status- noch prestigeträchtig ist oder hohes Einkommen verspricht, aber durchaus eine distinktive, gesellschaftskritische Deutung zulässt. Zudem ist zu vermuten, dass die passfähige habitusspezifische Distanzierung von einem wettbewerbsorientierten Leistungsverständnis bzw. von erwerbsorientiertem Karrierestreben zwar einerseits im linken Bereich des Raums der sozialen Milieus häufiger zu finden ist, aber auch gerade bei Frauen, deren Zuständigkeit milieuübergreifend weniger im Bereich der Erwerbsarbeit liegt als die von Männern. Mit Blick auf die Bedeutung von Vorbildern orientiert sich so auch Tabea an ihrer Mutter, die vor dem Hintergrund ihrer sozialarbeiterischen Tätigkeit eine Vorbildfunktion einnimmt. 


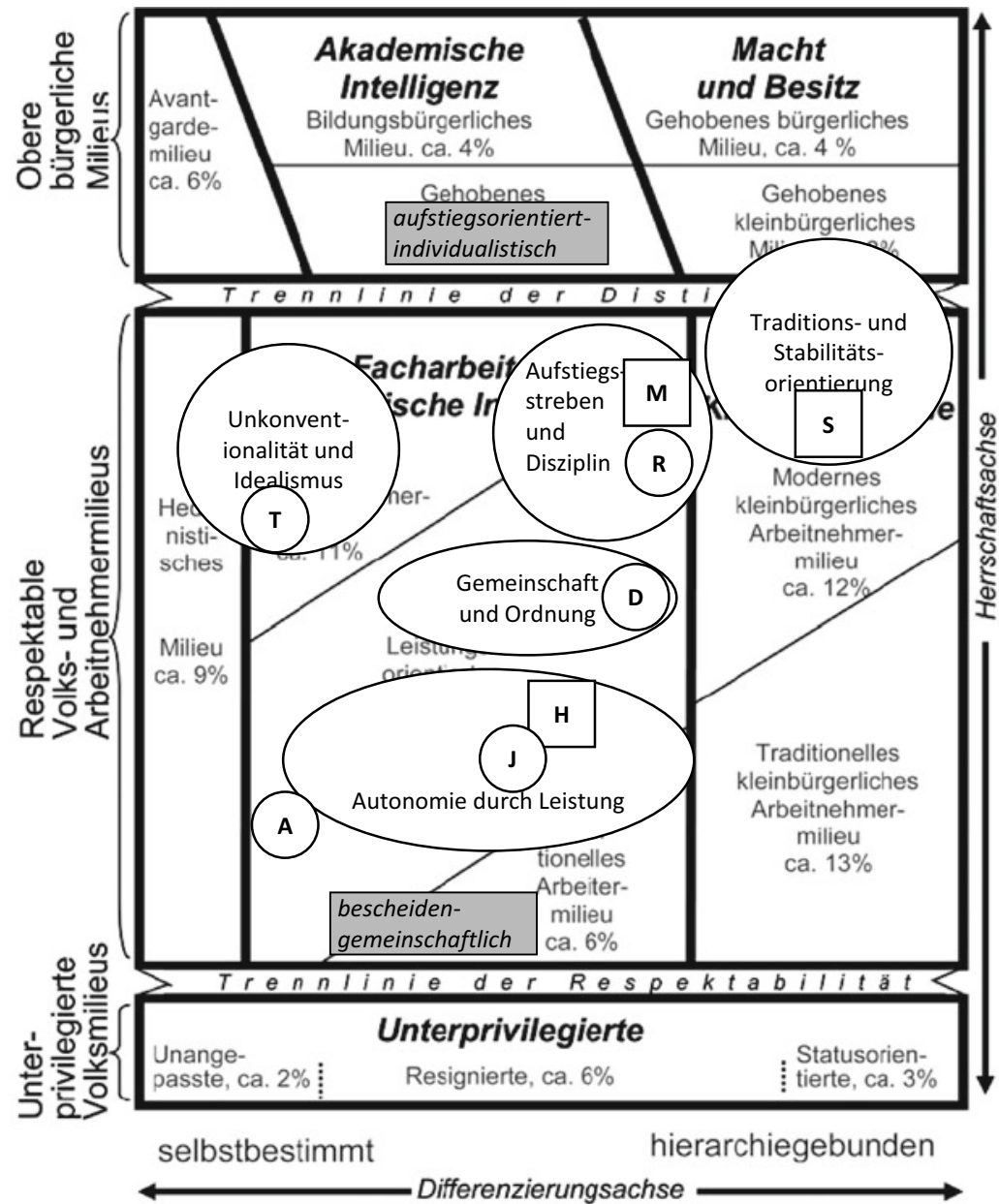

Abbildung 7.1 Muster der Studien(fach)wahl im untersuchten Sample und ihre Verortung im Modell sozialer Milieus. Erläuterung: Die Einzelfälle des Samples sind in geclusterten Mustern der Studien(fach)wahl zusammengefasst (,Unkonventionalität und Idealismus“, „Aufstiegsstreben und Disziplin“, „Traditions- und Stabilitätsorientierung“, „Gemeinschaft und Ordnung“ und „Autonomie durch Leistung“). Diese Muster sind spezifisch für den sozialen Ort der Befragten. Die Strukturierung der Muster wird zusätzlich durch die Pole ,aufstiegsorientiert-individualistisch“ (größte Nähe: „,M“ und „R“) und „bescheiden-gemeinschaftlich“ (größte Nähe: „A“, „,“, „,H“) verdeutlicht. Eine eigene Logik für Studien(fach)wahlen in der ständisch-kleinbürgerlichen Traditionslinie zeichnet sich zwar ab; ein eigener Pol kann hieraus jedoch nicht geschlossen werden, da in diesem Bereich nur ein Fall verortet ist (,S“). (Quelle: eigene Darstellung) 


\subsubsection{Studien(fach)wahlen im Kontext von Aufstiegsstreben und Disziplin}

Mit ihren vielen Parallelen lassen sich Michaels und Rebeccas habitusspezifische Muster der Studien(fach)wahl zusammenfassen unter dem gemeinsamen Nenner von „Aufstiegsstreben und Disziplin“. Hier zeigen sich an vielen Stellen Kontraste zum Muster „Unkonventionalität und Idealismus“, denn es spielen die statussichernde Funktion eines Berufs, sein gesellschaftliches Ansehen, ein wettbewerbsorientiertes Leistungsverständnis, Disziplin sowie ein besonderer Fokus auf das Erwerbsleben eine zentrale Rolle in den studien(fach)wahlbezogenen Habitusmustern. Auch materielle Werte und ein prätentiöses Vorgehen kommen hier vor. Die Familiengründung wird vor diesem Hintergrund - je nach Vergeschlechtlichung in unterschiedlicher Intensität - abhängig gemacht von bzw. ausgerichtet an der Erwerbsarbeit und teilweise kommt es zur Abwertung von Familienarbeit, was zu einem Spannungspotential auf weiblicher Seite führt.

Bei Rebecca spielen ebendiese Grundmuster des Habitus zusammen mit (familiären) Aufstiegsversuchen und Rebeccas prätentiösem ,Blick nach oben', im Rahmen dessen sie die Soziale Arbeit aufwertend deutet und in die Nähe statusträchtigerer Berufe rückt. Der Kontakt zur Sozialen Arbeit wurde in ihrem Fall durch ihre krisenhaften biografischen Erfahrungen hergestellt, die - zielgerichtet gefördert durch ihre Eltern - ihr Vertrauen in ihr eigenes Leistungspotential gestärkt und ihr Berufsziel der Kinder- und Jugendtherapeutin geprägt haben - ein Ziel, das für sie auch mit einer gewissen Vorstellung von Macht verbunden ist. Der Umgang der Eltern mit Rebeccas Schullaufbahn verweist ebenfalls auf eine individualistische Leistungsorientierung, den tragenden Wert von Disziplin, die Orientierung an einem höchst möglichen Schulabschluss und Rebeccas Anpassung in ein wettbewerbsorientiertes Leistungssystem. Ihrem Aufstiegswunsch entsprechend ist die Soziale Arbeit, so Rebeccas Plan, nur eine Zwischenstation zu ihrem Traumberuf als Therapeutin. Dass Rebeccas Leistungsorientierung stark erwerbsbezogen ist, schlägt sich auch in ihren Vorstellungen von Familiengründung nieder, denn Familienarbeit wertet sie $a b$ - antizipiert aber zugleich die Zuständigkeit dafür. So deutet sich ein Spannungspotential an zwischen Rebeccas versuchter Aufstiegsbewegung und erstens ihren milieuspezifischen Grenzen ,nach oben hin' sowie zweitens einer vergeschlechtlichten Abdrängung durch ebendiese Zuständigkeit für Familienarbeit auf weiblicher Seite. Fraglich ist, wie hoch in diesem Fall die kulturelle Passung zur sozialpädagogischen Fachkultur ist: Diese ist geprägt von einem personenzentrierten Lernverständnis jenseits konkurrenzbetonten Leistungsdenkens. Lernprobleme werden nicht individuellen Schwächen zugeschrieben, sondern der fehlenden Kompetenz der Lehrenden 
(Zinnecker 2004: 540). Rebeccas individualisierende Perspektive ist hieran nur bedingt anschlussfähig. Die fachkulturelle Haltung „Wettbewerb zerstört Solidarität" (Bargel 2007a: 199) ist bei ihr nicht zu erkennen, stattdessen schlägt sich bei Rebecca das Verständnis der Sozialen Arbeit als wettbewerbsfördernde Instanz nieder. So verdeutlicht ihr Fall, dass sich die Studierendenschaft in der Sozialen Arbeit keinesfalls auf eine dominante Ausprägung der Fachkultur (Bargel 2007a; Zinnecker 2004) beschränkt.

Bei Michaels Weg in das Studium schlagen sich ähnliche Grundmuster von Leistungsorientierung und Disziplin nieder, allerdings unter anderem mit dem Unterschied, dass er sich auch maßgeblich an materiellen Werten orientiert und seine Studien(fach)wahl in eine tendenziell patriarchal organisierte Herkunftsfamilie eingebettet ist. Zudem zeigt sich bei ihm weniger als bei Rebecca die Notwendigkeit zum ,Kampf' und weniger Anstrengung, was auf eine höhere vertikale Position und einen stärker abgesicherten familiären Status hindeutet. Michaels Weg lässt außerdem eine Verlagerung der familiären Strategien erahnen, vom Pol von Macht und Besitz stärker hin zur Kumulation von bildungsbezogenem kulturellem Kapital mit dem Ziel der Statusabsicherung. Eine fachliche Prägung hin zur Technik spiegelt sich in seiner Familie nicht wider: Den Einstieg in ein bautechnisches Berufsfeld macht er durch seine institutionell vermittelte Ausbildung zum Maurermeister, die Grundlage für sein Studium des Bauingenieurwesens ist und zu seinen Plänen der gemeinsamen Selbstständigkeit mit seiner Partnerin passt - die wiederum Architektur studiert. Auch Michaels Vorstellungen von Familiengründung sind an Erwerbsarbeit ausgerichtet, aber noch stärker als bei Rebecca, denn er macht die Familiengründung von seinem Erfolg im Beruf abhängig und antizipiert keinerlei Erziehungszeit auf seiner Seite. Damit ergibt sich für ihn wiederum kein Spannungspotential zwischen Erwerbs- und Familienarbeit.

Der Vergleich von Michael und Rebecca deutet zudem darauf hin, dass sich die milieuspezifischen Orientierungen an Leistung, Disziplin, Prestige und Erwerbsarbeit geschlechtsspezifisch auf die Studienfachwahl auswirken und mit unterschiedlichen Praktiken von Familienarbeit verbunden sind: So ist Michaels Erwerbsorientierung eine noch stärkere und ausschließlichere als bei Rebecca, die sich zudem nicht mit dem familiären Auftrag der Statusabsicherung konfrontiert sieht, sondern ebenso Zuständigkeit für die Familienarbeit antizipiert. Vor diesem Hintergrund wird Michael die „männliche Herrschaft“ , aufgezwungen“ (Bourdieu 2005: 195) (und er ist zur selbigen privilegiert), sodass eine soziale Studienfachwahl bei ihm äußerst unwahrscheinlich wäre, während Rebecca ihre milieuspezifische (und vergleichsweise bescheidene) Aufstiegs- und Statusorientierung auch durch eine Umdeutung der Sozialen Arbeit erfüllen kann. Beide 
Fälle zeichnet zudem aus, dass ihre berufsbezogene Orientierung - trotz Vorhandenseins weiblicher Bezugspersonen im Erwerbsleben - auf männlichen Bezugspersonen ausgerichtet ist (Väter, Partner), die für das Aufstiegsstreben eine bessere Orientierungsfläche zu bieten scheinen.

\subsubsection{Studien(fach)wahlen im Kontext von Gemeinschaft und Ordnung}

Das Muster der Studien(fach)wahl, das Dominics Fall prägt, lässt sich unter den zentralen Werten von „Gemeinschaft und Ordnung“ fassen. Das gemeinschaftliche Grundmuster von Dominics Habitus äußert sich in der hohen Bedeutung von Ratschlägen aus seinem sozialen Umfeld, von gemeinschaftlichen Entscheidungsprozessen auf Augenhöhe - die sich auch beim elterlichen Umgang mit ihm abzeichnen - von Freundschaften, familiären Bindungen und von emotionalem Rückhalt. Die Kombination, dass auch bei ihm weder Prestige noch materielle Werte handlungsleitend sind und dass er von seiner Gymnasial- auf eine Fachoberschullaufbahn gewechselt ist und dabei von seinen Eltern ressourcenorientiert unterstützt wurde, öffnet so seinen Weg in die Soziale Arbeit. Dabei kritisiert er die ,Ellbogenmentalität' seines ehemaligen Gymnasiums und ist dagegen an der Fachoberschule „,begeistert“, „,dass jeder Lehrer drauf geachtet hat, dass kein Schüler hintenrunter fällt". Dieses Positionierung gegenüber den beiden Schulsystemen verweist auf Dominics Nähe zur sozialpädagogischen Fachkultur, in der sich ein Lehrenden-Lernenden-Verhältnis auf Augenhöhe niederschlägt, in der die Lehrenden als „gute Hirten“ (Zinnecker 2004: 540) auch für die Schwächsten der Lerngruppe sorgen und in der gesellschaftlicher Wettbewerb häufig kritisch hinterfragt wird (Bargel 2007a: 199).

Zugleich verweist der Stellenwert von Anerkennung und teilweise Macht auf Anteile eines ständisch-kleinbürgerlichen Habitus: Dominic sieht seine künftigen Klient*innen als „Projekt“, das er bearbeiten und dann erfolgreich entlassen will. Er möchte wieder Ordnung in ihre Lebenswelt bringen, ähnlich einem ,Freund und Helfer'. Dieser Aspekt von Ordnung und Kontrolle schlägt sich auch in einem ursprünglichen Berufswunsch der Polizei nieder.

Dass die Gemeinschaft in diesem Muster der Studien(fach)wahl handlungsleitend ist, spiegelt sich auch in den Vorstellungen von Familiengründung wider: Auch hier werden der Zusammenhalt und die gemeinsame Zeit betont. Dies ist eine weitere Parallele zu den Erkenntnissen der Fachkulturforschung: Im Rahmen der von Haffner (2014) festgestellten fachkulturspezifischen Männlichkeitskonstruktionen antizipieren die Studenten der Sozialen Arbeit eine vergleichsweise 
starke Involvierung in die spätere Erziehungsarbeit. Dies ist auch bei Dominic der Fall und eng verwoben mit seinen milieuspezifischen Dispositionen.

\subsubsection{Studien(fach)wahlen im Kontext von Traditions- und Stabilitätsorientierung}

Sonjas Habitus verweist auf die Grundmuster der ständisch-kleinbürgerlichen Traditionslinie und soll im Kontext ihrer Studien(fach)wahl unter dem Titel von „Traditions- und Stabilitätsorientierung“ zusammengefasst werden. Bezeichnend dafür sind Dispositionen hin zu klaren Strukturen, zu Ordnung und Bekanntem, die in ihrem Fall einhergehen mit einer fachlichen Affinität zu ,Logik' und einer Anlehnung an die familiäre Tradition der technischen Studienfachwahl, die auch die weiblichen Berufswahlen umfasst. So resultiert ein geradliniger, strategischer und geplanter Weg mit großer Selbstverständlichkeit hin zum Studium, aber auch mit vergeschlechtlichten Abdrängungseffekten. Diese betreffen sowohl die vergleichsweisen bescheidenen Wege der Frauen in Sonjas Familie, die ihr Studium an Fachhochschulen statt Universitäten absolvieren, wie auch Sonjas teilweise negative Unterrichtserfahrungen. Zudem ist die Familienorganisation von einer weiblichen Zuständigkeit für die Familienarbeit geprägt: Was Sonjas Vorstellungen von Familiengründung betrifft, nimmt diese erstens einen großen Stellenwert ein und beinhaltet zweitens die schwerpunktmäßige Ausübung der Erziehungsarbeit durch Sonja selbst.

Zum familiären Erbe der technischen Studienorientierung gehört auch die Weitergabe eines Selbstbildes über die ,Sonderrolle als Frau': So erwartet Sonja einen geringen Frauenanteil im Studium sowie Vereinbarkeitsprobleme von Beruf und Familie und sie nimmt rückblickend in ihrer Schulzeit geschlechtsspezifische Benachteiligungen wahr. Trotzdem verfolgt sie ihr mathematisches Fachinteresse und wählt ein Studium hin zu einem Ingenieurberuf - denn ihr familiärer Hintergrund, ihre hohe Passung zur ingenieuralen Fachkultur mit ihren logischen Inhalten und dem ,nüchternen Anwendungsbezug“ (Sander/Weckerth 2017) und ihre milieuspezifischen Orientierungen an Tradition und Stabilität stärken sie bei diesem Weg. Die matriarchale Weitergabe des Wissens über die ,Sonderrolle als Frau' wiederum kann vor diesem Hintergrund als strategischer Vorteil gelesen werden, durch den Sonja einige ,Spielregeln' technischer Berufsfelder kennt und ihre Erwartungen daran ausrichten kann. Zusätzlich deuten ihre berufsbezogenen Bewertungs- und Handlungsmuster nicht darauf hin, dass sie sich gegen diese Spielregeln auflehnen will oder diese im Widerspruch zu ihren Vorstellungen von 
Familien- und Erwerbsarbeit stünden. Vielmehr ist eine Einordnung in die vergeschlechtlichte Struktur ihres künftigen Berufsfeldes ist vor dem Hintergrund von Sonjas ständisch-kleinbürgerlichem Habitus zu vermuten.

Und obwohl Sonjas Fall selbst im vertikal-mittleren Bereich des Milieumodells zu verorten ist, weil sich keine ausgeprägten Anzeichen von Distinktion zeigen und sie durchaus mit Unsicherheiten zu kämpfen hat, ist eine grundsätzliche Überlappung des Studien(fach)wahlmusters von „Traditions- und Stabilitätsorientierung" mit dem oberen Bereich der privilegierten Milieus aufgrund der Selbstverständlichkeit des Studiums und des hohen Anteils an studienbezogenen Ressourcen wahrscheinlich.

\subsubsection{Studien(fach)wahlen im Kontext von Autonomie durch Leistung}

Das letzte Muster von „Autonomie durch Leistung“ umfasst die Fälle von Jennifer und Hanna, deren Muster der Studien(fach)wahl durch einen Bildungsaufstieg geprägt sind, der mit Anstrengung genommen wird und auf Unabhängigkeit und Autonomie abzielt ${ }^{6}$. Zugleich gibt es innerhalb dieses Musters auch einige Unterschiede, die vor allem auf die in den Herkunftsfamilie vorhandenen Dispositionen und beruflichen Hintergründe zurückzuführen sind.

Jennifers bildungsbezogener Aufstieg und ihr Weg in die Soziale Arbeit sind nicht nur von der Orientierung an Autonomie, sondern auch von ihren Erfahrungen des Unsteten und der Notwendigkeit geprägt, von ihrer frühen familiären Verantwortungsübernahme und ihrem fürsorglich geprägten Selbstbild. Die soziale Gemeinschaft bedeutet für sie Sicherheit und prägt stark ihre berufs- und studienfachwahlbezogenen Entscheidungen. Mit ihrer familiären Verwurzelung im Dienstleistungsmilieu mündet sie in eine Büroausbildung ein und vollzieht den anschließenden Bildungsaufstieg gemeinsam mit und unterstützt durch ihren Partner, der ebenfalls aus einer Familie ohne Hochschulerfahrung stammt. Trotz ihrer Risikobereitschaft schlägt sich bei Jennifers Vorstellungen von Familiengründung ihre finanzielle Sicherheitsorientierung nieder, weshalb sie eine traditionelle Arbeitsteilung antizipiert.

\footnotetext{
${ }^{6}$ Einen Bildungsaufstieg findet man auch bei Achim wieder, bei dem ebenfalls die Orientierung an Autonomie eine Rolle spielt - deshalb wird sein Fall in entsprechender Nähe zu diesem Muster verortet. Bei den anderen Fällen ohne akademischen familiären Hintergrund (Michael, Dominic) zeichnen sich wiederum eher horizontale Verlagerungen ab als ein tatsächlicher (vertikaler) Bildungsaufstieg.
} 
Auch Hannas bildungsbezogener Aufstieg von dem Streben nach Autonomie, insbesondere nach beruflicher Handlungsfreiheit geprägt: Verwurzelt im Traditionellen Arbeitermilieu beginnt sie ihren Bildungsaufstieg über eine technische Ausbildung und gemeinsam mit ihrer Zwillingsschwester. Ihre latente Orientierung an sozialen Hierarchien, in denen sie durch Leistung aufsteigen will, und ihre Eingebundenheit in ihr Herkunftsdorf, die auch bei Hanna mit einem gemeinschaftlichen Habituszug verknüpft ist, prägen ebenso ihren Weg in das Studium und ihre Berufsvorstellungen. Ihre Vorstellungen von Familiengründung sind abgesehen davon, dass sie eine Familie gründen will - noch offen. Im Gegensatz zu Jennifer scheint sich Hanna stärker den Normen ihrer Herkunftsfamilie entgegensetzen zu müssen, wobei beide auf nur wenig familiäre Ressourcen für das Studium zurückgreifen können.

Zusammengenommen ist der Weg in das Studium allgemein beim Muster von „Autonomie durch Leistung“ mit einer (teilweisen) Abkehr von den herkunftsspezifischen Habitusmustern verbunden, mit einer gewissen Risikobereitschaft und mit dem entscheidenden Einfluss gleichgesinnter Aufsteiger*innen ${ }^{7}$. In diesem Kontext knüpfen die Fachwahlen für ein soziales oder technisches Studium zum einen an die fachlichen Prägungen in der Herkunftsfamilie bzw. dem Kreis von Peers an und ebenso an die milieuspezifischen Erfahrungen und Orientierungen sodass im Ergebnis beide Fachwahlen einen ähnlichen milieuspezifischen Zweck erfüllen, nämlich Autonomie und beruflichen Aufstieg.

\section{Fall Achim: Autonomiestreben jenseits ,typischer' Milieuorientierungen}

Der Fall von Achim soll aufgrund seiner ausgeprägten Orientierung an Autonomie mit Nähe zum Muster von „Autonomie durch Leistung“ dargestellt werden. Gleichzeitig spiegeln Achims Habituszüge nur teilweise die Spezifika des Leistungsorientierten Arbeitnehmermilieus wider. Zudem zeichnen sich auch Hinweise auf das Hedonistische Milieu bei ihm ab. Seine spezifischen Erfahrungen und Dispositionen lassen keine schlüssige Milieuhypothese zu. Vielleicht ist es eben das, was diesen Fall eines männlichen Sozialarbeitsstudenten (und möglicherweise auch andere ,männliche ' Wege in die Soziale Arbeit) prägt.

In Achims habitusspezifischen Studien(fach)wahlmustern spielt vor diesem Hintergrund zusammen, dass er von seinem sozialen Umfeld offen und gemeinschaftlich unterstützt wurde jenseits fester (Bildungs- oder Berufs-)Ziele, dass er Erfahrungen finanzieller Begrenzung gemacht hat und dass seine Mutter keine

\footnotetext{
${ }^{7}$ Dieser Zusammenhang erinnert an die Bedeutung von ,sozialen Paten“ (Mafaalani 2014) für den Bildungsaufstieg, also die Unterstützung durch Menschen aus höheren Milieus wobei bei den Bildungsaufstiegen im hier vorliegenden Sample der gemeinsame Aufstieg mit Menschen ähnlicher bzw. gleicher sozialer Herkunft der entscheidende Einfluss ist.
} 
Erwerbserfahrung, aber einen breiten Hintergrund in unbezahlten Fürsorgetätigkeiten hat und seine zentrale Bezugsperson ist. Dadurch ist Sorgearbeit für Achim schon früh positiv besetzt und er hat bereits während der Schulzeit berufliche Erfahrungen mit der Jugendarbeit gemacht. Seinen Berufswunsch, ein Jugendzentrum zu leiten, hat er so bereits als Jugendlicher entwickelt und daran hält er ,verbissen“ fest. Zugleich geht er jedoch spontan und flexibel vor in seinem Weg dorthin, hält sich die Alternativen von Jura und Geophysik offen. Allgemein ist Achims Lebensplanung eine eher mittelfristige, bei der er sich auf lange Sicht nicht festlegen will. Das zeigt sich auch in seinen Vorstellungen von Familiengründung: Auch diese ist für ihn lediglich eine Option.

Diese Option findet allerdings vor dem Hintergrund einer positiven Orientierung statt, denn er sieht sich als künftiger ,guter Papa“, was erneut auf die Erkenntnisse von Haffner (2014) zu den eher egalitären, beteiligenden Familienvorstellungen von Sozialarbeitsstudenten verweist. Im Vergleich zu den anderen beiden männlichen Fällen von Michael und Dominic deutet sich so an, dass die von Haffner festgestellten fachkulturspezifischen Männlichkeitskonstruktionen in sozialen und technischen Studiengängen zugleich auf milieuspezifische Männlichkeitskonstruktionen verweisen: Während bei Achim sowie im Muster von „Gemeinschaft und Ordnung" eine männlich-vergeschlechtlichte Nähe zu grundsätzlich offenen Familienvorstellungen, zu einer tendenziell (!) egalitären Aufgabenteilung ${ }^{8}$, zur Mit-Übernahme von Erziehungstätigkeiten und zum hohen Wert gemeinschaftlicher Familienzeit festzustellen ist, zeigt sich beim Muster von „Aufstiegsstreben und Disziplin“ eine ausgeprägte Erwerbsorientierung und die Abgrenzung von Erziehungsaufgaben.

\subsection{Fokus Soziale Arbeit: Änderung der Gesellschaft, Fitmachen für den Wettbewerb oder Herstellen von Ordnung}

Die Überschrift greift drei beispielhafte, recht kontrastive milieuspezifische Sichtweisen auf die gesellschaftliche Funktion der Sozialen Arbeit auf, die sich im Sample abbilden. Sie verdeutlichen, wie unterschiedlich der Weg in dieses Studium gefärbt sein kann, je nach Habitualisierung in einem bestimmten sozialen

\footnotetext{
${ }^{8}$ Es kann hier tatsächlich nur von Tendenzen gesprochen werden, denn eine egalitäre Aufgabenteilung antizipiert keine der befragten Personen und entsprechende Geschlechterpraktiken zeichnen sich weder auf manifester noch latenter Ebene des Materials $\mathrm{ab}$.
} 
Milieu. Dabei zeichnen sich in einigen Fällen durchaus Anteile einer dominanten sozialpädagogischen Fachkultur ab (Bargel 2007a; Zinnecker 2004; Haffner 2014), aber zugleich eröffnet sich ein Blick auf vielfältige Zugänge zur Sozialen Arbeit innerhalb der Studierendenschaft.

So ist kennzeichnend für den sozialen Ort von Tabea, dass sie die Soziale Arbeit in idealistischer Weise als gesellschaftsformende Instanz hin zu mehr Egalität betrachtet und sich dabei Anteile eines distinktiven Vorgehens abzeichnen. Wettbewerbsorientiertes Karrierestreben wertet sie ab und möchte ihre Zeit lieber dem ,große[n] Ganzen“ widmen, das sie in der Sozialen Arbeit sieht dem Zwischenmenschlichen und Gemeinschaftlichen. Konträr dazu steht der Fall von Rebecca: Sie deutet die Soziale Arbeit in einem individualistischen Leistungsverständnis, in dessen Rahmen sie zur „Umstellung“ und „Erziehung“ ihrer Klient*innen beitragen will. Der*die Einzelne soll fitgemacht werden für den gesellschaftlichen Wettbewerb, so wie Rebecca selbst dafür erfolgreich fitgemacht wurde. Ihr ausgeprägtes Aufstiegsstreben führt wiederum dazu, dass sie ihre berufliche Zukunft in der sendungsbewussten Position einer Kinder- und Jugendtherapeutin sieht und die Soziale Arbeit nur eine Zwischenstation dorthin sein soll. Zugleich zeichnen sich bei Rebecca Werte von Ordnung und Autorität ab, die jedoch bei Dominic noch stärker hervortreten: Zwar ist auch sein Fall (wie der von Tabea) geprägt von gemeinschaftlichen und kooperativen Habituszügen, allerdings sieht er seine Klient*innen als „Projekt“ und er möchte ihre aus den Fugen geratene Lebenswelt wieder, in Ordnung' bringen. Der Wert von Gemeinschaft wie auch der Blick auf die Soziale Arbeit in ihrer Ordnungsfunktion prägen Dominics Weg in das Studium ebenso wie seinen ursprünglichen Berufswunsch, Polizist zu werden. Bezeichnenderweise schlägt sich dieser alternative Berufswunsch mit seiner kontrollierenden, ordnenden gesellschaftlichen Funktion auch bei Rebecca nieder, die zudem juristischen Inhalten nahe ist.

Die weiteren Fälle von Jennifer und Achim wiederum verweisen auf ebenjenen Aspekt, der das verbindende Element zwischen vielen - nicht allen - Wegen in die Soziale Arbeit ist und auch die Nähe zur entsprechenden Fachkultur maßgeblich prägt: Der Wert von Gemeinschaft, kooperative Handlungsmuster und ein besonderer Fokus auf zwischenmenschliche Beziehungen. Wie wichtig Freundschaften sind, dass Entscheidungen gemeinsam mit dem sozialen Umfeld getroffen werden und dass sorgende, erzieherische und/oder pflegerische Tätigkeiten positiv besetzt und wertvoll sind, prägt diese Fälle. Diese Disposition hin zu Fürsorge wird in Jennifers Fall matriarchal weitergegeben, in Achims Fall dagegen eher im Rahmen egalitärer Geschlechterpraktiken. Zugleich zeichnet sich eine gewisse Bedeutung von Notwendigkeitserfahrungen ab, durch die die Gemeinschaft erst an besonderem Wert gewinnt und materieller Besitz Sicherheit bedeutet und mit Bescheidenheit verbunden ist. 
Zusammengenommen ist die antizipierte Nähe zur sozialpädagogischen besonders ausgeprägt in jenen Fällen, deren Habitus von gemeinschaftlichen, kooperativen Handlungsmustern, von einer Distanzierung von Prestige- und Karrierestreben oder auch einer gewissen Ordnungsorientierung geprägt sind. Jenseits davon lassen aber auch stärker individualistische, konkurrenzorientierte Handlungsmuster eine (möglicherweise geringer ausgeprägte) fachkulturelle Nähe $\mathrm{zu}$ - zumindest in einem, weiblichen' Fall.

\subsection{Fokus Bauingenieurwesen: Affinität zur Logik, Erfolg im Business oder Schritt zum beruflichen Aufstieg}

Auch hier verweist die Überschrift auf jene Aspekte, die die im Sample abgebildeten milieuspezifischen Wege in das Bauingenieurwesen prägen. So kann das Bauingenieurwesen an unterschiedliche habitusspezifische Dispositionen anknüpfen, deren Schwerpunkte sich jedoch von denen unterscheiden, die einen Weg in die Soziale Arbeit ebnen. Auch hier spiegeln sich Elemente der ingenieuralen Fachkultur (Sander/Weckerth 2017) in den analysierten Passungsverhältnissen wider, die sich darin zugleich nicht erschöpfen.

Der soziale Ort von Sonja eröffnet ihr die grundsätzliche Sicherheit und Selbstverständlichkeit, ein Studium aufzunehmen und die fachlichen Dispositionen ihrer Herkunftsfamilie fördern ihre Nähe zur ingenieuralen Fachkultur. Verstärkt wird dies durch Sonjas Orientierung hin zu Bekanntem, zu übersichtlichen Strukturen und einer entsprechenden Affinität zu eindeutigen, ,logischen * Fachinhalten. In diesem Rahmen und von zahlreichen Akademiker*innen in technischen Berufen umgeben entwickelt auch Sonja früh eine fachliche Nähe zu mathematischen Inhalten, die sie in das praxisnahe, überschaubare Bauingenieurwesen an einer Hochschule für Angewandte Wissenschaft führt. Vergleichweise weniger fachliche Nähe - insbesondere keine familial angelegte - findet sich im Fall von Michael: Hier ist es vielmehr das Potential des Bauingenieurwesens als erfolgreiches Geschäft, das die Studien(fach)wahl maßgeblich prägt. Die institutionell gerahmte Vermittlung in die Maurerausbildung ist Michaels erster Schritt in die Baubranche, in der er sich später selbstständig machen und Immobilienhandel betreiben will - ähnlich wie er jetzt den Autohandel aus seiner Herkunftsfamilie kennt. Seine Orientierung an Disziplin, Exklusivität und beruflichem Aufstieg komplettieren das Bild von seinem Weg in das Studium. Eine ausgeprägte fachliche Nähe wiederum rückt bei Hanna wieder in den Vordergrund: Ihre Habitualisierung ist von einer grundsätzlichen Distanz zu einer 
akademischen Ausbildung geprägt, allerdings von einem frühen Kontakt zur Baubranche über ihren Vater, wodurch ihr fachliches Interesse geweckt und der Weg in das Studium des Bauingenieurwesens geebnet wird. Treibende Kraft in ihrem Schritt aus der Ausbildung zur Bauzeichnerin heraus hin zur Hochschule ist ihr Autonomiestreben und ihr Wunsch nach Aufstieg in der beruflichen Hierarchie.

Den unterschiedlichen Vorstellungen zum Trotz verbindet alle Ingenieursfälle geschlechtsübergreifend ihre grundsätzlich hohe (Hanna) bis sehr hohe (Sonja und Michael) Passung zur technischen Fachkultur (Sander/Weckerth 2017), die auf einer Fokussierung auf Erwerbsarbeit und Beruf, auf materiellen Werten, gewissen Ansprüche an Einkommen, auf dem nüchternen Anwendungsbezug und/oder auf dem beruflichen Aufstieg fußt.

Ingesamt bildet sich im Sample eine schwerpunktmäßige Verortung der Fälle von Bauingenieurstudierenden weiter ,rechts' und weiter , oben ' im Raum der sozialen Milieus als die der Sozialen Arbeit. Dies ist ein Hinweis auf eine grundsätzliche Tendenz der milieuspezifischen Anschlussfähigkeit der entsprechenden Habitusmuster an die jeweilige Fach- und Berufskultur. Gleichzeitig wurden jedoch ebenso große Schnittmengen deutlich und es wurde gezeigt, dass die milieuspezifischen Perspektiven auf die beiden Studiengänge und Berufsfelder von Bauingenieurwesen und Sozialer Arbeit äußerst heterogen sind.

\subsection{Fokus Geschlecht: der Wert von Gemeinschaft und die Kraft von Vorbildern}

Was das Geschlecht betrifft, scheinen es bestimmte habitusspezifische Erfahrungen und Muster zu sein, die die geschlechtlich, erwartungswidrigen' Wege in ein technisches bzw. soziales Studium bestärken. So verbindet die männlichen Sozialarbeitsstudenten die ausgeprägte Orientierung an Gemeinschaft, die bei den weiblichen Fällen nicht immer vorhanden ist. Das Zusammenspiel von Gemeinschaftsorientierung, der Abgrenzung von Statusorientierung und wettbewerbsorientiertem Leistungsdenken sowie eine gewisse Offenheit gegenüber zumindest tendenziell egalitären Geschlechterpraktiken scheint ein Türöffner für Männer zu sein, Sozialarbeiter zu werden. Diese Dispositionen und Habituszüge finden sich tendenziell im Raum sozialer Milieus horizontal tendenziell links und mittig, während die schwerpunktmäßige vertikale Verortung im mittleren Bereich bis hin zur Grenze der Respektabilität zu vermuten ist. Eine gewisse Anschlussfähigkeit ist jedoch auch über der Grenze der Distinktion denkbar.

Unter den weiblichen Bauingenieurstudentinnen wiederum zeichnen sich weniger gemeinsame Habituszüge ab, die diese Fachwahl entscheidend begünstigen. 
Die Studentinnen sind jedoch verbunden durch ähnlich gelagerte Erfahrungen, nämlich den Einblick in technische Berufe über das soziale Umfeld und die dadurch geförderte Affinität zu technisch-mathematischen Fach- und Berufsinhalten. Diese fachliche Ausrichtung wiederum kann durch unterschiedliche milieuspezifische Orientierungen flankiert und bestärkt werden - wie jene an Tradition und Stabilität oder jene an Autonomie und beruflichem Aufstieg. Die Vorstellungen von Familiengründung und -organisation scheinen diese Wege weniger zu beeinflussen als bei den männlichen Sozialarbeitsstudenten - denn im Fall der Bauingenieurstudentinnen bedeutet eine Studien(fach)wahl jenseits binärer Zuschreibungen nicht zwangsläufig eine egalitär ausgerichtete Vorstellung von Familiengründung.

Was die geschlechtlich ,erwartungskonformen" Studien(fach)wahlen von Frauen für die Soziale Arbeit und von Männern für das Bauingenieurwesen betrifft, erlauben die vorgenommenen Habitus- und Milieuanalysen einen Blick hinter fachlich-binäre Zuschreibungen im Spiegel von ,Technik' und ,Sozialem'. Der, weibliche' Blick auf die Soziale Arbeit könnte etwa bei Tabea und Rebecca nicht unterschiedlicher sein, umfasst er einmal das Berufsverständnis als egalitäre und gesellschaftsformende und einmal als erziehende und wettbewerbsfördernde Instanz. Bezeichnend ist hier der Widerspruch von Rebeccas erwerbsorientiertem Aufstiegsstreben und ihre vergleichsweise prestige- und statusarme Fachwahl sowie ihre Antizipation der von ihr abgewerteten Erziehungsarbeit. Dies mag ein Hinweis darauf sein, dass diese Kombination bei einem ein ,männlichen ' Fall vergleichbarer sozialer Position und mit ähnlichen Habitusmustern nicht denkbar wäre. Dass wiederum die Fachwahl hin zur Sozialen Arbeit eben auch mit der sozialen Vererbung weiblicher Zuständigkeit für Fürsorge einhergehen kann, zeigt der Fall von Jennifer: Ihre frühen fürsorglichen Erfahrungen als Unterstützung ihrer Mutter in der Erziehung ihrer Geschwister machen ihre hohe Passung zur Sozialen Arbeit maßgeblich aus. Der Fall von Michael letztendlich verdeutlicht, dass die Verknüpfung von ,Männlichkeit' und ,Technik' im Sinne einer fachkulturellen Sozialisation nicht entscheidend für eine ingenieurale Fachwahl von Männern sein muss - denn hier ist vielmehr die dahinter liegende Verknüpfung von ,Männlichkeit‘ und Erwerbszuständigkeit bzw. Aufstiegsstreben die maßgebliche. 
Open Access Dieses Kapitel wird unter der Creative Commons Namensnennung 4.0 International Lizenz (http://creativecommons.org/licenses/by/4.0/deed.de) veröffentlicht, welche die Nutzung, Vervielfältigung, Bearbeitung, Verbreitung und Wiedergabe in jeglichem Medium und Format erlaubt, sofern Sie den/die ursprünglichen Autor(en) und die Quelle ordnungsgemäß nennen, einen Link zur Creative Commons Lizenz beifügen und angeben, ob Änderungen vorgenommen wurden.

Die in diesem Kapitel enthaltenen Bilder und sonstiges Drittmaterial unterliegen ebenfalls der genannten Creative Commons Lizenz, sofern sich aus der Abbildungslegende nichts anderes ergibt. Sofern das betreffende Material nicht unter der genannten Creative Commons Lizenz steht und die betreffende Handlung nicht nach gesetzlichen Vorschriften erlaubt ist, ist für die oben aufgeführten Weiterverwendungen des Materials die Einwilligung des jeweiligen Rechteinhabers einzuholen.

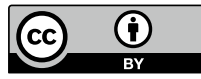

